\title{
PERIODIC SOLUTIONS OF STOCHASTIC DELAY DIFFERENTIAL EQUATIONS AND APPLICATIONS TO LOGISTIC EQUATION AND NEURAL NETWORKS
}

\author{
Dingshi Li And DaOYi Xu
}

\begin{abstract}
In this paper, we consider a class of periodic Itô stochastic delay differential equations by using the properties of periodic Markov processes, and some sufficient conditions for the existence of periodic solution of the delay equations are given. These existence theorems improve the results obtained by Itô et al. [6], Bainov et al. [1] and Xu et al. [15]. As applications, we study the existence of periodic solution of periodic stochastic logistic equation and periodic stochastic neural networks with infinite delays, respectively. The theorem for the existence of periodic solution of periodic stochastic logistic equation improve the result obtained by Jiang et al. [7].
\end{abstract}

\section{Introduction}

Since Itô introduced his stochastic calculus about 50 years ago, the theory of stochastic differential equations has been developed very quickly $[1-15,17]$. It is now being recognized to be not only richer than the corresponding theory of differential equations without stochastic perturbation but also represent a more natural framework for mathematical modeling of many real-world phenomena. Now there also exists a well-developed qualitative theory of stochastic differential equations $[6,10,12]$. However, not so much has been developed in the direction of the periodically stochastic differential equations. Till now only a few papers have been published on this topic $[1,3,4,15,17]$. In papers $[3,6]$, the authors got the conditions for the existence of periodic solution of differential equations with random right sides. Hasminskii in [4] gave some basic results on the existence of periodic solution of stochastic differential equations without delays. But, the above results can not be used to check the existence of periodic solution of general stochastic delay differential equations. In [15], Xu et

Received March 24, 2011; Revised May 6, 2013.

2010 Mathematics Subject Classification. 34A37, 34D23.

Key words and phrases. stochastic differential equations, periodic solution, infinite delay, logistic equation, neural networks.

The work is supported by National Natural Science Foundation of China under Grant 11271270 and 11201320. 
al. showed that stochastic differential equations with finite delay (SFDE) has a periodic solution if its solutions are uniformly bounded and point dissipativity. Motivated by the above discussions, we will generalize the existence theorem of the periodic solution for SFDE to stochastic differential equations with infinite delay (ISFDE) at phase space $B C\left((-\infty, 0] ; R^{n}\right)$. The obtained results improve the results obtained by Itô et al. [6], Bainov et al. [1] and $\mathrm{Xu}$ et al. [15]. As applications, we study the existence of periodic solution of periodic stochastic logistic equation and periodic stochastic neural networks with infinite delays, respectively. The theorem for the existence of periodic solution of periodic stochastic logistic equation improve the result obtained by Jiang et al. [7]

\section{Preliminaries}

For convenience, we introduce several notations and recall some basic definitions.

$C[X, Y]$ denotes the space of continuous mappings from the topological space $X$ to the topological space $Y$. Let $R_{+}=(0,+\infty)$ and $\bar{R}_{+}=[0,+\infty)$. Especially, let $B C \triangleq C\left([-\tau, 0], R^{n}\right)$ is the space of all bounded continuous $R^{n}$-value functions $\phi$ defined on $[-\tau, 0]$ with the norm $\|\phi\|=\sup _{-\tau \leq s \leq 0}|\phi(s)|$, where $|\cdot|$ is any norm in $R^{n}$ and $\tau$ is a fixed number or $\tau=\infty$. When $\tau=\infty$ we mean, of course, that $B C \triangleq C\left((-\infty, 0], R^{n}\right)$. Let $\left(\Omega, \mathscr{F},\left\{\mathscr{F}_{t}\right\}_{t \geq 0}, P\right)$ be a complete probability space with a filtration $\left\{\mathscr{F}_{t}\right\}_{t \geq 0}$ satisfying the usual conditions (i.e., it is right continuous and $\mathscr{F}_{0}$ contains all $P$-null sets). If $x(t)$ is an $R^{n}$ valued stochastic process on $t \in[-\tau, \infty)$, we let $x_{t}=x(t+s):-\tau \leq s \leq 0$, which is regarded as a $B C$-valued stochastic process for $t \geq 0$. Denote by $B C_{\mathscr{F}_{0}}^{b}\left([-\tau, 0], R^{n}\right)$ the family of all bounded $\mathscr{F}_{0}$-measurable, $B C$-valued random variables $\phi$, satisfying $E[\|\phi\|]<\infty$, where $E[f]$ mean the mathematical expectation of $f$.

Definition 2.1. A stochastic process $x_{t}(\varpi)$ with values in Banach space $B C$, defined for $t \geq 0$ on a probability space $\left(\Omega, \mathscr{F},\left\{\mathscr{F}_{t}\right\}_{t \geq 0}, P\right)$ is called a Markov process if, for all $A \in \mathscr{B}, 0 \leq v<t$,

$$
P\left\{x_{t}(\varpi) \in A \mid \mathscr{F}_{v}\right\}=P\left\{x_{t}(\varpi) \in A \mid x_{v}(\varpi)\right\},
$$

where $\mathscr{F}_{v}$ is the $\sigma$-algebra of events generated by all events of the form $\left\{x_{u}(\varpi)\right.$ $\in A, u \leq v\}$ and $\mathscr{B}$ denotes the $\sigma$-algebra of Borel sets in $B C$.

Definition 2.2. A stochastic process $x_{t}(\varpi)$ is said to be periodic with period $\omega$ if its finite dimensional distributions are periodic with periodic $\omega$, i.e., for any positive integer $m$ and any moments of time $t_{1}, \ldots, t_{m}$, the joint distributions of the random variables $x_{t_{1+k \omega}}(\varpi), \ldots, x_{t_{m+k \omega}}(\varpi)$ are independent of $k(k=$ $\pm 1, \pm 2, \ldots)$.

Remark 2.1. By the definition of periodicity, if $x(t)$ is a $\omega$-periodic stochastic process, then its mathematic expectation and variance are $\omega$-periodic [4, p. 49]. 
The transition function of a Markov process, $p\left(v, x_{v}, t, A\right)=P\left(x_{t} \in A \mid x_{v}\right)$, a.s., is called periodic if $p\left(v, x_{v}, t+v, A\right)$ is periodic in $v$.

Later on we shall often denote a family of Markov processes by $x_{t}^{\left(t_{0}, \phi\right)}(\varpi)$ for all $t_{0} \in R_{+}$and $x_{t_{0}}=x\left(t_{0}+s\right)=\phi(s) \in B C_{\mathscr{F}_{0}}^{b}\left([-\tau, 0], R^{n}\right)$.

Definition 2.3. The Markov families $x_{t}^{\left(t_{0}, \phi\right)}(\varpi)$ are said to be uniformly bounded, if for each $\alpha>0, t_{0} \in R_{+}$, there exists a positive constant $\theta=\theta(\alpha)$ which is independent of $t_{0}$ such that $E\left\|x_{t_{0}}\right\| \leq \alpha$ implies $E\left[\left\|x_{t}\left(t_{0}, x_{t_{0}}\right)\right\|\right] \leq \theta$, $t \geq t_{0}$. In a general way, the Markov families $x_{t}^{\left(t_{0}, \phi\right)}(\varpi)$ are said to be $p$ uniformly bounded if $E[\|\cdot\|]$ is replaced by $E\left[\|\cdot\|^{p}\right]$.

Denote $U_{r}=\{\phi \in B C:\|\phi\|<r\}$ by $\bar{U}_{r}=\{\phi \in B C:\|\phi\| \geq r\}$.

Lemma 2.1. A sufficient condition for the existence of an $\omega$-periodic Markov process with a given $\omega$-periodic transition function $p\left(v, x_{v}, t, A\right)$ is that for some $t_{0}, \phi, x_{t}^{\left(t_{0}, \phi\right)}(\varpi)$ are uniformly stochastically continuous and

$$
\lim _{r \rightarrow \infty} \frac{\varliminf_{T \rightarrow \infty}}{T} \frac{1}{T} \int_{t_{0}}^{t_{0}+T} p\left(t_{0}, \phi, t, \bar{U}_{r}\right) d t=0,
$$

provided the transition function $p\left(v, x_{v}, t, A\right)$ satisfies the following not very restrictive assumption that

$$
\alpha(r)=\sup _{\phi \in U_{\beta(r)}, 0<t_{0}, t-t_{0}<\omega} p\left(t_{0}, \phi, t, \bar{U}_{r}\right) \rightarrow 0 \quad \text { as } r \rightarrow \infty,
$$

for some function $\beta(r)$ which tends to infinity as $r \rightarrow \infty$.

The proof of Lemma 2.1 is essentially the same as that of Lemma 2.3 in [15], Theorem 2.1 including Remark 2.1 in [1] and [9, Theorem 2.1, p. 491] except one chooses $\phi \in B C_{\mathscr{F}_{0}}^{b}\left([-\tau, 0], R^{n}\right)$. But, in fact, the conditions of Lemma 2.1 are of little use for stochastic differential equations, since the properties of transition functions of such processes are usually not expressible in terms of the coefficients of the equation. So, in the following, we will give some new useful sufficient conditions.

Lemma 2.2. If Markov families $x_{t}^{\left(t_{0}, \phi\right)}(\varpi)$ with $\omega$-periodic transition functions are uniformly bounded and uniformly stochastically continuous, then there is an $\omega$-periodic Markov process.

Proof. Using Markov inequality, Xu et al. [15, p. 1009] gave that

$$
p\left(t_{0}, \phi, t, \bar{U}_{r}\right) \leq \frac{1}{r P\left(x_{t_{0}}=\phi\right)} E\left[\left\|x_{t}\right\|\right] .
$$

From the definition of uniform boundedness, for each $\alpha>0$, there exists a positive constant $\theta=\theta(\alpha)$ such that $E\|\phi\| \leq \alpha$ implies $E\left[\left\|x_{t}\left(t_{0}, x_{t_{0}}\right)\right\|\right] \leq \theta$, $t \geq t_{0}$. 
So, we get

$$
\begin{aligned}
& \lim _{r \rightarrow \infty} \frac{\varliminf_{T \rightarrow \infty}}{T} \int_{t_{0}}^{t_{0}+T} p\left(t_{0}, \phi, t, \bar{U}_{r}\right) d t \\
\leq & \lim _{r \rightarrow \infty} \frac{1}{r P(\phi)} \underline{\varliminf_{T \rightarrow \infty}} \frac{1}{T} \int_{t_{0}}^{t_{0}+T} E\left[\left\|x_{t}\right\|\right] d t \\
\leq & \lim _{r \rightarrow \infty} \frac{\theta(\alpha)}{r P(\phi)}=0,
\end{aligned}
$$

that is, (1) is true. The proof of (2) is similar to the remainder of the proof of Theorem 2.4 in [15], so we omit it.

Lemma $2.3([16])$. If

$\left(A_{1}\right) u: R_{+} \rightarrow R^{n}$ is uniformly continuous,

$\left(A_{2}\right) g: R^{n} \rightarrow R$ is continuous and $g(x)=0$ if and only if $x=0$,

$\left(A_{3}\right) h: R_{+} \rightarrow R_{+}$satisfies $\inf _{t \geq 0} \int_{t}^{t+\delta} h(s) d s>0$ for any $\delta>0$,

$\left(A_{4}\right) \lim _{t \rightarrow \infty} \int_{0}^{t} h(s) g(u(s)) d s$ exists and is finite.

Then $\lim _{t \rightarrow \infty} u(t)=0$.

\section{Periodic solution of ISFDE}

In this section, we consider the following periodic ISFDE

$$
\left\{\begin{array}{l}
d x(t)=f\left(t, x_{t}\right) d t+g\left(t, x_{t}\right) d W(t), t \geq t_{0} \geq 0 \\
x_{t_{0}}=x\left(t_{0}+s\right)=\phi(s), s \in[-\tau, 0]
\end{array}\right.
$$

on the probability space $\left(\Omega, \mathscr{F},\left\{\mathscr{F}_{t}\right\}_{t \geq 0}, P\right)$. The equation (3) has a Borel measurable $\omega$-periodic drift coefficient function $f:\left[t_{0}, \infty\right) \times B C \rightarrow R^{n}$ and a Borel measurable $\omega$-periodic diffusion coefficient function $g:\left[t_{0}, \infty\right) \times B C \rightarrow$ $R^{n \times m}$ driven by $m$-dimensional Brownian motion $W$. We assume that $\mathscr{F}_{t}$ is the completion of the $\sigma$-algebra $\sigma\left\{W(u): t_{0} \leq u \leq t\right\}$ for each $t \geq t_{0}$. The initial condition $\phi(s) \in B C_{\mathscr{F}_{0}}^{b}\left([-\tau, 0], R^{n}\right)$ is independent of $W(t), t \geq t_{0}$, and $E[\|\phi\|]<\infty$.

In this section, we always assume that system (3) has a unique global solution (see $[13,14])$.

Theorem 3.1. Assume that the solutions of periodic system (3) are p-uniformly bounded for $p>2$ and $f\left(t, x_{t}\right)$ and $g\left(t, x_{t}\right)$ satisfy

$$
\left|f\left(t, x_{t}\right)\right|^{p}+\left|g\left(t, x_{t}\right)\right|^{p} \leq \varphi\left(\left\|x_{t}\right\|^{p}\right), \quad p>2,
$$

where $\varphi$ is a concave non-decreasing function, then there is an $\omega$-periodic Markov process.

Proof. As the proof of Lemma 3.2 in [15], we have that the unique solution $x_{t}$ of $(3)$ is a Markov process with its transition function $p\left(v, x_{v}, t, A\right)=$ $P\left(x_{t} \in A \mid x_{v}\right)$. Moreover, the transition function $p\left(v, x_{v}, t, A\right)$ of $(3)$ is $\omega$ periodic since the coefficients of $(3)$ are $\omega$-periodic in $t$. 
Since $\varphi$ is a concave non-decreasing function, we get

$$
E \varphi\left(\left\|x_{t}\right\|^{p}\right) \leq \varphi\left(E\left\|x_{t}\right\|^{p}\right) .
$$

From the $p$-boundedness of $x_{t}$ and the condition (4), we can get that there exists a constant $\mu>0$ such that

$$
E\left|f\left(t, x_{t}\right)\right|^{p}+E\left|g\left(t, x_{t}\right)\right|^{p} \leq \mu, \quad p>2 .
$$

By Lemma 3.4 in [15], the solutions of periodic system (3) are uniformly stochastically continuous.

In the view of above, all conditions of Lemma 2.2 are satisfied. The proof is complete.

Suppose (3) is the autonomous system, that is, $f\left(t, x_{t}\right)$ and $g\left(t, x_{t}\right)$ do not depend on $\mathrm{t}$. We have the following corollary:

Corollary 3.1. Assume that the solutions of autonomous system (3) are puniformly bounded for $p>2$ and $f\left(x_{t}\right)$ and $g\left(x_{t}\right)$ satisfy Condition (4), then there is a stationary Markov process.

Remark 3.1. Itô et al. [6, Theorem 2, p. 19] (Bainov et al. [1, Theorem 5.3, p. 23]) gave that there is a stationary (periodic) Markov process of autonomous (periodic) system (3) if the uniformly bounded in 4-th moment and

$$
\left\{\begin{array}{l}
\left|f\left(t, x_{t}\right)\right|^{4} \leq q_{1}+\int_{-\infty}^{0}|x(s)|^{4} d k_{1}(s), \\
\left|g\left(t, x_{t}\right)\right|^{4} \leq q_{2}+\int_{-\infty}^{0}|x(s)|^{4} d k_{2}(s),
\end{array}\right.
$$

where $q_{1}, q_{2}$ are some positive constants and $k_{i}(s), i=1,2$, are scalar nondecreasing bounded functions on $(-\infty, 0]$. Clearly, Conditions $(5)$ is a special case of Conditions (4) by taking $p=4$ and $\varphi$ to be linear. Therefore, Corollary 3.1 and Theorem 3.1 is a generation of Th.2 in [6] and Th.5.3 in [1], respectively.

Let $V \in C^{1,2}\left([-\tau,+\infty) \times B C, \bar{R}_{+}\right)$denote the family of all non-negative real-value functions $V\left(t, x_{t}\right)$ on $[-\tau,+\infty) \times B C$ which are continuously twice differentiable in the first term and once in the second term. If $V \in C^{1,2}([-\tau,+\infty)$ $\left.\times B C, \bar{R}_{+}\right)$, define an operator $L V$ from $[-\tau,+\infty) \times B C$ to $R$ by

$$
L V\left(t, x_{t}\right)=V_{t}\left(t, x_{t}\right)+V_{x}\left(t, x_{t}\right) f\left(t, x_{t}\right)+\frac{1}{2} \operatorname{trace}\left[g^{T}\left(t, x_{t}\right) V_{x x}\left(t, x_{t}\right) g\left(t, x_{t}\right)\right],
$$

where $V_{t}\left(t, x_{t}\right)=\frac{\partial V\left(t, x_{t}\right)}{\partial t}, V_{x}\left(t, x_{t}\right)=\left(\frac{\partial V\left(t, x_{t}\right)}{\partial x_{1}}, \ldots, \frac{\partial V\left(t, x_{t}\right)}{\partial x_{n}}\right), V_{x x}\left(t, x_{t}\right)=$ $\left(\frac{\partial^{2} V\left(t, x_{t}\right)}{\partial x_{i} \partial x_{j}}\right)_{n \times n}$.

Theorem 3.2. Assume that the solutions of (3) are uniformly stochastically continuous. Let $V \in C^{1,2}\left([-\tau,+\infty) \times B C, \bar{R}_{+}\right)$and satisfy that there exists a constant $M$ such that

$$
L V\left(t, x_{t}\right) \leq 0, \quad\left\|x_{t}\right\| \geq M
$$




$$
\inf _{\left\|x_{t}\right\| \in \bar{U}_{R}} V\left(t, x_{t}\right) \rightarrow \infty \quad \text { as } \quad R \rightarrow \infty .
$$

Then Eq.(3) has a w-periodic solution.

Proof. Without loss of generality, fix the initial value $x_{t_{0}} \in \bar{U}_{M}$ arbitrarily. We write $x_{t}=x_{t}\left(t_{0}, x_{t_{0}}\right)$ and set $V_{r}=\inf _{x_{t} \in \bar{U}_{r}} V\left(t, x_{t}\right)$, where $r>M$. Let $\tau$ be the first enter time of $x_{t}$ into $U_{M}$, that is

$$
\tau=\inf \left\{t \geq t_{0}: x_{t} \in U_{M}\right\} .
$$

By Itô's formula, for any $t \geq t_{0}$,

$$
\begin{aligned}
V\left(\tau \wedge t, x_{\tau \wedge t}\right)= & V\left(t_{0}, x_{t_{0}}\right)+\int_{t_{0}}^{\tau \wedge t} L V\left(s, x_{s}\right) d s \\
& +\int_{t_{0}}^{\tau \wedge t} V_{x}\left(s, x_{s}\right) g\left(s, x_{s}\right) d W(s) .
\end{aligned}
$$

Taking the expectation on both sides and making use of the condition (6), we obtain that

$$
E V\left(\tau \wedge t, x_{\tau \wedge t}\right) \leq V\left(t_{0}, x_{t_{0}}\right) .
$$

Using this and Čebyšev's inequality, we get

$$
\begin{aligned}
P\left(\left\|x_{t}\right\| \geq r\right) & \leq \frac{E V\left(\tau \wedge t, x_{\tau \wedge t}\right)}{V_{r}} \\
& \leq \frac{V\left(t_{0}, x_{t_{0}}\right)}{V_{r}} .
\end{aligned}
$$

This yields

$$
\begin{aligned}
p\left(t_{0}, \phi, t, \bar{U}_{r}\right) & =P\left(x_{t} \in \bar{U}_{r} \mid x_{t_{0}}=\phi\right) \\
& =\frac{P\left\{\left(\left\|x_{t}\right\| \geq r\right) \cap\left(x_{t_{0}}=\phi\right)\right\}}{P\left(x_{t_{0}}=\phi\right)} \\
& \leq \frac{P\left(\left(\left\|x_{t}\right\| \geq r\right)\right)}{P\left(x_{t_{0}}=\phi\right)} \rightarrow 0 \quad \text { as } r \rightarrow \infty .
\end{aligned}
$$

Clearly, the condition (1) of Lemma (2.1) holds.

Further, it follows from the condition (6) that $L V\left(t, x_{t}\right) \leq K, x_{t} \in B C$, where $K$ is a sufficiently large constant. Using this inequality and Itô's formula, we easily obtain that

$$
E V\left(t, x_{t}\right) \leq V\left(t_{0}, x_{t_{0}}\right)+K\left(t-t_{0}\right) .
$$

Together with Čebyšev's inequality, this implies

$$
p\left(t_{0}, x_{t_{0}}, t, \bar{U}_{R}\right) \leq \frac{K\left(t-t_{0}\right)+V\left(t_{0}, x_{t_{0}}\right)}{\inf _{\left\|x_{t}\right\| \in \bar{U}_{R}} V\left(t, x_{t}\right)} .
$$


Thus the condition (2) of Lemma 2.1 will hold if $\beta(R)$ is chosen so that

$$
\frac{\sup _{\left\|x_{t}\right\| \in U_{\beta(R)}} V\left(t, x_{t}\right)}{\inf _{\left\|x_{t}\right\| \in \bar{U}_{R}} V\left(t, x_{t}\right)} \rightarrow 0 \text { as } R \rightarrow \infty .
$$

This is possible because (7) holds. The proof is completed.

Remark 3.2. The uniform stochastic continuity is required to guarantee the compactness of stochastic sequences defined a infinite dimensional space $B C$. For the stochastic ODE defined the finite dimensional space $R^{n}$, the uniform stochastic continuity is not required. Therefore, we have the following corollary.

Corollary 3.2. Assume that system (3) without delays exists the global solutions. Let $V \in C^{1,2}\left(\bar{R}_{+} \times R^{n}, \bar{R}_{+}\right)$and satisfy that there exists a constant $M$ such that

$$
\begin{gathered}
L V(t, x) \leq 0, \quad|x| \geq M, \\
\inf _{|x|>R} V(t, x) \rightarrow \infty \quad \text { as } \quad R \rightarrow \infty .
\end{gathered}
$$

Then Eq.(3) without delays has a $\omega$-periodic solution.

\section{Periodic solution of nonautonomous stochastic logistic equation}

In this section, we consider the following nonautonomous stochastic logistic equation [7]

$$
d N(t)=N(t)[a(t)-b(t) N(t)] d t+\sigma(t) N(t) d B(t),
$$

on $t \geq 0$ with initial value $N(0)=N_{0}>0$, and $a(t), b(t)$ and $\sigma(t)$ are continuous functions. $B(t)$ is a Brownian motion defined on $\left(\Omega, \mathscr{F},\left\{\mathscr{F}_{t}\right\}_{t \geq 0}, P\right)$.

As $N(t)$ in Eq.(11) is population size at time $t$, it should be nonnegative. Moreover, in order for a stochastic differential equation to have a unique global (i.e., no explosion in a finite time) solution for any given initial data, the coefficients of the equation are generally required to satisfy the linear growth condition and local Lipschitz condition. However, the coefficients of Eq.(11) do not satisfy the linear growth condition, though they are locally Lipschitz continuous, so the solution of Eq.(11) may explode at a finite time. In this section we shall show that under simple hypothesis the solution of Eq.(11) is not only positive but will also not explode at any finite time by using the global existence theorem in $[13,14]$.

Theorem 4.1. Assume

(H1) There exist constants $\sigma_{1}, \sigma_{2}, a_{1}, a_{2}, b_{1}>0, b_{2}>0$ and continuous bounded function $h(t) \geq 0$ such that

$$
\sigma_{1} h(t) \leq \sigma^{2}(t) \leq \sigma_{2} h(t), a_{1} h(t) \leq a(t) \leq a_{2} h(t), b_{1} h(t) \leq b(t) \leq b_{2} h(t) .
$$


Then for any initial conditions $N_{0}>0$, there is a unique solution $x(t)$ to Eq.(11) on $t \geq 0$, and the solution will remain in $R_{+}$almost surely, i.e., $x(t) \in$ $R_{+}$for any $t \geq 0$ with probability 1 .

Proof. First consider the equation

$$
d u(t)=\left(a(t)-b(t) e^{u(t)}-\frac{1}{2} \sigma^{2}(t)\right) d t+\sigma(t) d B(t)
$$

on $t \geq 0$ with initial value $u(0)=\ln N(0)$. Obviously, the coefficients of Eq.(12) satisfy the local Lipschitz condition, then there is a unique non-continuable solution $u(t)$ on $t \in\left[0, \tau_{e}\right)$, where $\tau_{e}$ is the right endpoint of the maximum existing interval of $u(t)$ (see $[13,14]$ ). Therefore, by Itô's formula, it is easy to see $N(t)=e^{u(t)}$, is the unique positive solution to Eq.(11) with initial value $N(0) \in R_{+}$.

Next, we will show this non-continuable solution is global, i.e., $\tau_{e}=\infty$, a.s. To show this statement, let us define a $C^{1,2}$-function $V: \bar{R}_{+} \rightarrow \bar{R}_{+}$by

$$
V(N)=[\sqrt{N}-1-0.5 \log (N)] .
$$

The nonnegativity of this function can be seen from

$$
\sqrt{u}-1-0.5 \log (u) \geq 0, \quad u>0 .
$$

Note that

$$
\lim _{N \rightarrow \infty} V(N)=\infty .
$$

Moreover, we estimate operator $L V$. Since

$$
\begin{aligned}
L V(N)= & -0.5 b(t) N^{1.5}+0.5 b(t) N+0.5 a(t) N^{0.5}-0.5 a(t) \\
& +0.25 \sigma^{2}(t)-0.125 \sigma^{2}(t) N^{0.5}
\end{aligned}
$$

by hypothesis $(H 1)$, we obtain

$$
\begin{aligned}
& L V(N) \\
\leq & \left(-0.5 b_{1} N^{1.5}+0.5 b_{2} N+0.5 a_{2} N^{0.5}-0.5 a_{1}-0.125 \sigma_{1}^{2} N^{0.5}+0.25 \sigma_{2}^{2}\right) h(t) .
\end{aligned}
$$

Since the coefficient of highest-degree term of the right side is negative and $h(t)$ is bounded, there is a constant $K>0$ such that

$$
E L V(x) \leq K
$$

It follows from Theorem 5.1 in [14] that $\tau_{e}=\infty$, a.s. The proof is complete.

Theorem 4.2. Assume that $a(t), b(t)$ and $\sigma(t)$ are continuous $T$-periodic functions, $(H 1)$ and

(H2) $B=\int_{0}^{T}\left[a(s)-\frac{1}{2} \sigma^{2}(s)\right] d s>0$

hold. Then Eq.(11) has a positive T-periodic solution. Moreover, if

(H3) $\inf _{t \geq 0} \int_{t}^{t+\delta} h(s) d s>0$, for any $\delta>0$ 
hold. Then Eq.(11) has a unique positive T-periodic solution, which attracts all other positive solutions of Eq.(11).

Proof. From Theorem 4.1, we know that for any initial conditions $N_{0} \in R_{+}$, there is a unique positive solution $N(t)$ to Eq.(11) on $t \geq 0$ under Condition (H1). Taking the transformation of the form

$$
u=\ln N \text {. }
$$

By Itô's formula, we have

$$
d u(t)=\left(a(t)-b(t) e^{u(t)}-\frac{1}{2} \sigma^{2}(t)\right) d t+\sigma(t) d B(t)
$$

on $t \geq 0$ with initial value $u(0)=\ln N(0)$.

For any initial value $u(0) \in R$, we denote by $u(t)=u(t, 0, u(0))$ the solution of (14). Define

$$
p(t)=-\int_{0}^{t}\left(a(s)-\frac{1}{2} \sigma^{2}(s)-B h(s)\right) d s,
$$

where $B$ is defined in $(H 2)$. It is to see that $p(t)$ is $T$-periodic continuous functions. Set

$$
v(t)=u(t)+p(t) .
$$

By Itô's formula, we have

$$
d v(t)=\left(B h(t)-b(t) e^{v(t)-p(t)}\right)+\sigma(t) d B(t) .
$$

For (15), we define a Lyapunov function

$$
V(v)=|v|,
$$

which satisfies (10).

Then we have

$$
L V(v)=\operatorname{sgn} v\left\{B h(t)-b(t) e^{v(t)-p(t)}\right\}
$$

Condition $(H 1)$ implies that

$$
L V(v) \leq h(t)\left(B-b_{1} e^{v(t)-p(t)}\right) \leq 0 \quad \text { as } \quad v \rightarrow \infty .
$$

We consider the case $v \rightarrow-\infty$. For the above $B>0$, there exists $l>0$ such that

$$
b_{2} e^{v(t)-p(t)}<B, \quad v<-l
$$

Thus,

$$
\begin{aligned}
L V(v) & =\left\{-B h(t)+b(t) e^{v(t)-p(t)}\right\} \\
& \leq h(t)\left(-B+b_{2} e^{v(t)-p(t)}\right) \leq 0 .
\end{aligned}
$$

From (16) and (18), we get that there exists a positive constant $M$ such that

$$
L V \leq 0, \quad|v| \geq M
$$


Thus, the condition (9) of Corollary 3.2 is satisfied, and there is a periodic solution of (14) or (15). From $N(t)=e^{u(t)}$, Eq.(11) has a positive periodic solution.

In order to prove the uniqueness of periodic solution $N^{*}(t)$ of Eq.(11), we will prove the global attractivity of $N^{*}(t)$ by Lemma 2.3. To this end, we need that sample path of the solution $\mathrm{N}(\mathrm{t})$ of Eq.(11) is uniformly continuous. This conclusion has been proved by Lemma 3.2 in [8] under $b(t)>0 \Rightarrow E N^{p}(t)<\infty$. So, we only need prove that $b(t) \geq 0$ with $(H 1) \Rightarrow E N^{p}(t)<\infty$. Define

$$
V(N(t))=e^{\int_{0}^{t} h(s) d s} N^{p}(t),
$$

where $p>0$. By Itô's formula, we have that

$$
\begin{aligned}
& d V(N(t))=e^{\int_{0}^{t} h(s) d s} N^{p}(t)\left[h(t)+p a(t)+\frac{1}{2} p(p-1) \sigma^{2}(t)-p b(t) N(t)\right] d t \\
& \quad+p e^{\int_{0}^{t} h(s) d s} N^{p}(t) d B(t) .
\end{aligned}
$$

Integrating (20) from 0 to $t$ and taking expectation on both sides, we obtain from $(H 1)$ that

$$
\begin{aligned}
& e^{\int_{0}^{t} h(s) d s} E N^{p}(t) \\
= & N^{p}(0)+E \int_{0}^{t} e^{\int_{0}^{s} h(u) d u} N^{p}(s)\left[h(s)+p a(s)+\frac{1}{2} p(p-1) \sigma^{2}(s)-p b(s) N(s)\right] d s \\
\leq & N^{p}(0)+E \int_{0}^{t} e^{\int_{0}^{s} h(u) d u} N^{p}(s) h(s)\left[1+p a_{2}+\frac{1}{2} p|p-1| \sigma_{2}-p b_{1} N(s)\right] d s \\
\leq & N^{p}(0)+K \int_{0}^{t} e^{\int_{0}^{s} h(u) d u} h(s) d s \\
= & N^{p}(0)+K\left(e^{\int_{0}^{t} h(s) d s}-1\right),
\end{aligned}
$$

where $K$ is the maximum of the function $N^{p}\left[1+p a_{2}+\frac{1}{2} p|p-1| \sigma_{2}-p b_{1} N\right]$. Then we have

$$
E N^{p}(t) \leq N^{p}(0)+K<\infty .
$$

Following Lemma 3.2 in [8], this yields that almost every sample path of the solution $N(t)$ of Eq.(11) is uniformly continuous on $t \geq 0$.

In order to apply Lemma 2.3, we consider a Lyapunov function $V(t)$ defined by

$$
V(t)=\left|\log N(t)-\log N^{*}(t)\right|, \quad t \geq 0 .
$$

By Itô's formula, we have that

$$
d\left(\log N(t)-\log N^{*}(t)\right)=-b(t)\left(N(t)-N^{*}(t)\right) d t .
$$

Thus, a direct calculation of the right differential $d^{+} V(t)$ of $V(t)$ along the solutions leads to

$$
\begin{aligned}
d^{+} V(t) & =\operatorname{sgn}\left(N(t)-N^{*}(t)\right) d\left(N(t)-N^{*}(t)\right) \\
& =-\operatorname{sgn}\left(N(t)-N^{*}(t)\right)\left[b(t)\left(N(t)-N^{*}(t)\right)\right] d t
\end{aligned}
$$


PERIODIC SOLUTIONS OF STOCHASTIC DELAY DIFFERENTIAL EQUATIONS 1175

$$
=-b(t)\left|N(t)-N^{*}(t)\right| d t
$$

Integrating (21) from 0 to $t$, we have

$$
V(t)+\int_{0}^{t} b(s)\left|N(s)-N^{*}(s)\right| d s \leq V(0)<\infty .
$$

which leads to

$$
\int_{0}^{\infty} b_{1} h(s)\left|N(s)-N^{*}(s)\right| d s \leq V(0) .
$$

Therefore from Lemma 2.3 and (H3), we obtain

$$
\lim _{t \rightarrow+\infty}\left|N(s)-N^{*}(s)\right|=0 \quad \text { for almost all } \omega \in \Omega,
$$

which shows that $N^{*}(t)$ attracts all other positive solutions of Eq.(11). This implies that Eq.(11) has a unique positive $T$-periodic solution.

Remark 4.1. In [7], the authors showed that $E[1 / N(t)]$ has a unique positive $T$-periodic solution $E\left[1 / N_{p}(t)\right]$ provided the condition $(H 2), a(t)>0$ and $b(t)>0$. From Theorem 4.2, Eq.(11) has a unique positive $T$-periodic solution. So is $1 / N_{p}(t)$ or $E\left[1 / N_{p}(t)\right]$ with periodic $\mathrm{T}$ by Remark 2.1. But, under the condition (H2) we need not $a(t)>0$ and admit $b(t) \geq 0$ with $(H 1)$ and (H3). The improvement is in effect since the usual periodic functions $\sin t, \cos t$ etc. are admitted. However, a benefit of the results in [7] is to get the representation of global solution.

\section{Application in the stochastic neural networks with infinite delays}

For convenience, we introduce several notations and recall some basic definitions. Let $I$ denote an $n \times n$ unit matrix. For $A, B \in R^{m \times n}$ or $A, B \in R^{n}$, the notation $A \geq B(A>B)$ means that each pair of corresponding elements of A and B satisfies the inequality " $\geq(>)$ ". Especially, $A \in R^{m \times n}$ is called a nonnegative matrix if $A \geq 0$, and $z \in R^{n}$ is called a positive vector if $z>0$.

$$
\wp=\left\{\psi(t): R_{+} \rightarrow R \mid \psi(t) \text { is continuous and } \int_{0}^{\infty} \psi(s) \mathrm{d} s<\infty\right\} .
$$

For $\varphi \in C\left[J \subset R, R^{n}\right]$, we define

$$
[\varphi(t)]_{\tau}=\left\{\left[\varphi_{1}(t)\right]_{\tau}, \ldots,\left[\varphi_{n}(t)\right]_{\tau}\right\}, \quad\left[\varphi_{i}(t)\right]_{\tau}=\sup _{-\tau \leq \theta \leq 0}\left\{\varphi_{i}(t+\theta)\right\} .
$$


In this section, the periodic stochastic neural networks with infinite delays is described by the following model

(23)

$$
\left\{\begin{aligned}
& d x_{i}(t)= {\left[-a_{i}(t) x_{i}(t)+\sum_{j=1}^{n} a_{i j}(t) f_{j}\left(x_{j}(t)\right)+\sum_{j=1}^{n} b_{i j}(t) \int_{-\infty}^{t} p_{i j}(t-s) g_{j}\left(x_{j}(s)\right) d s+I_{i}(t)\right] d t } \\
&+\sum_{j=1}^{n} \sigma_{i j}\left(t, x_{i}(t)\right) d w_{j}(t), \quad t \geq t_{0} \geq 0 \\
& x_{i}\left(t_{0}+s\right)=\phi_{i}(s), \quad s \in(-\infty, 0], \quad i=1,2, \ldots, n,
\end{aligned}\right.
$$

where $n$ corresponds to the number of units in a neural network; $x_{i}(t)$ corresponds to the state of the $i$ th unit at time $t ; f_{j}\left(x_{j}(t)\right)$ and $g_{j}\left(x_{j}(t)\right)$ denote the activation functions of the $j$ th unit at time $t ; a_{i}(t) \geq 0$ represents the rate with which the ith unit will reset its potential to the resting state in isolation when disconnected from the network and external inputs; $\left(a_{i j}(t)\right)_{n \times n}$ and $\left(b_{i j}(t)\right)_{n \times n}$ are connection matrices; the delay kernel $p_{i j}(t) \in \wp ; I_{i}(t)$ is the external bias on the ith unit; We assume that functions $a_{i}(t), a_{i j}(t), b_{i j}(t)$ and $I_{i}(t)$ are periodic continuous functions with periodic $\omega>0$ for $t \geq t_{0}, i, j=1, \ldots, n ; \sigma(\cdot, \cdot)=\left(\sigma_{1}(\cdot, \cdot), \ldots, \sigma_{n}(\cdot, \cdot)\right):\left[t_{0}, \infty\right) \times R^{n} \rightarrow$ $R^{n \times n}$ is the $\omega$-periodic diffusion coefficient matrix; $w(t)=\left(w_{1}(t), \ldots, w_{n}(t)\right)^{T}$ is an $n$-dimensional Brownian motion defined on $\left(\Omega, \mathscr{F},\left\{\mathscr{F}_{t}\right\}_{t \geq 0}, P\right)$. The initial condition $\phi(s) \in B C_{\mathscr{F}_{0}}^{b}\left((-\infty, 0], R^{n}\right)$.

For convenience, we will introduce the following assumptions.

$\left(A_{1}\right)$ All $f_{j}$ and $g_{j}$ satisfy the Lipschitz condition, that is, there exist $l_{j}>0$ and $k_{j}>0$ such that

$\left|f_{j}(u)-f_{j}(v)\right| \leq l_{j}|u-v|,\left|g_{j}(u)-g_{j}(v)\right| \leq k_{j}|u-v|, \forall u, v \in R, j=1, \ldots, n$.

$\left(A_{2}\right)$ There exist constants $c_{i} \geq 0, d_{i} \geq 0$ such that for $u_{1}, u_{2}, \in R, i=$ $1, \ldots, n$,

$$
\left|\left(\sigma_{i}\left(t, u_{1}\right)-\sigma_{i}\left(t, u_{2}\right)\right)\left(\sigma_{i}\left(t, u_{1}\right)-\sigma_{i}\left(t, u_{2}\right)\right)^{T}\right| \leq c_{i} h(t)\left|u_{1}-u_{2}\right|^{2} .
$$

where $h(t) \geq 0$ and $\sup _{0<t<\infty} h(t)<\infty$.

$\left(A_{3}\right)$ There exist constants $a_{i}, a_{i j} \geq 0, b_{i j} \geq 0, I_{i}>0$ such that

$$
\begin{aligned}
& a_{i}(t) \geq a_{i} h(t), \quad\left|a_{i j}(t)\right| \leq a_{i j} h(t), \quad\left|b_{i j}(t)\right| \leq b_{i j} h(t), \\
& \left|I_{i}(t)\right| \leq I_{1} h(t), \quad \forall i, j=1,2, \ldots, n .
\end{aligned}
$$

$\left(A_{4}\right)$ There exists an integral $p>2$ such that $S=-(P+Q)$ is an $M$-matrix, where $P=\left(p_{i j}\right)_{n \times n}, Q=\left(q_{i j}\right)_{n \times n}$,

$$
\begin{aligned}
p_{i i}= & -p a_{i}+\sum_{j=1}^{n} a_{i j} l_{j}(p-1)+\sum_{j=1}^{n} b_{i j} k_{j}(p-1) \int_{0}^{\infty}\left|p_{i j}(s)\right| d s+(p-1) \\
& +\frac{1}{2} c_{i} p(p-1)+a_{i i} l_{i}, \\
p_{i j}= & a_{i j} l_{j}, \quad i \neq j, \quad q_{i j}=b_{i j} k_{j} \int_{0}^{\infty}\left|p_{i j}(s)\right| d s .
\end{aligned}
$$


For the sake of simplicity, we use $x(t)$ to denote the solutions $x\left(t_{0}, \phi\right)(t)$ of (23). In order to obtain the boundedness of the stochastic system (23), we first show the following theorem.

Theorem 5.1. Let $P=\left(p_{i j}\right)_{n \times n}$ with $p_{i j} \geq 0(i \neq j)$ and $Q(t)=\left(q_{i j}(t)\right)_{n \times n}$ with $0 \leq q_{i j}(t) \in \wp$. Denote $Q=\left(q_{i j}\right)_{n \times n} \triangleq\left(\int_{0}^{\infty} q_{i j}(t) \mathrm{d} t\right)_{n \times n}$ and $I=$ $\left(I_{1}, \ldots, I_{n}\right)>0$. Let $D=-(P+Q)$ be an $M$-matrix, and $u(t)=\left(u_{1}(t), u_{2}(t)\right.$, $\left.\ldots, u_{n}(t)\right)^{T}$ a nonnegative continuous vector function satisfies the following differential inequality

$$
D^{+} u(t) \leq h(t)\left[P u(t)+\int_{0}^{\infty} Q(s) u(t-s) \mathrm{d} s+I\right], \quad t \geq t_{0},
$$

with the initial condition $u\left(t_{0}+s\right)=\phi(s) \in B C^{+}, s \in(-\infty, 0]$, where $B C^{+}=$ $\{\phi \in B C \mid \phi \geq 0\}$ and $h(t) \geq 0$, then all solutions of the inequality (24) are uniformly bounded.

Proof. Since $D=-(P+Q)$ is an $M$-matrix, from the properties of $M$-matrix [16, Lemma 2.1], $D^{-1} I>0$. For any given initial function $\phi \in B C^{+}$, there is a $d \geq 0$ such that $\phi \leq-d(P+Q)^{-1} I$. We will prove that

$$
u(t) \leq-d(P+Q)^{-1} I, \quad t \geq t_{0},
$$

that is, all solutions of the inequality (24) are uniformly bounded.

We set that $-(P+Q)^{-1} I=N$. So, we have $(P+Q) N+I=0$, or,

$$
\sum_{j=1}^{n} p_{i j} N_{j}+\sum_{j=1}^{n} q_{i j} N_{j}+I_{i}=0, \quad i=1,2, \ldots, n .
$$

If (25) is not true, then there must exist a positive constant $t_{1}>t_{0}$ and some integer $m$ such that

$$
\begin{array}{r}
u_{m}\left(t_{1}\right)=d N_{m}, \quad D^{+} u_{m}\left(t_{1}\right)>0, \\
u_{i}(t) \leq d N_{i}, \quad t \in\left(-\infty, t_{1}\right], \quad i=1,2, \ldots, n .
\end{array}
$$

By using (24), (25), (26), (28) and $p_{i j} \geq 0(i \neq j), q_{i j} \geq 0$, noting $d \geq 1$, we have

$$
\begin{aligned}
D^{+} u_{m}\left(t_{1}\right) & \leq\left[\sum_{j=1}^{n} p_{m j} u_{j}\left(t_{1}\right)+\sum_{j=1}^{n} q_{m j}\left[u_{j}\left(t_{1}\right)\right]_{\infty}+I_{i}\right] h(t) \\
& \leq\left[\sum_{j=1}^{n}\left(p_{m j}+q_{m j}\right) d N_{j}+I_{i}\right] h(t) \\
& =(1-d) I_{m} h(t) \leq 0,
\end{aligned}
$$

which contradicts the inequality in (25). That implies that $u(t)$ is uniformly bounded. 
Theorem 5.2. Suppose that $\left(A_{1}\right)-\left(A_{4}\right)$ hold, then the system (23) must have a $\omega$-periodic Markov process.

Proof. Let $V_{i}(x(t))=\left|x_{i}(t)\right|^{p}, i=1, \ldots, n$, where $x(t)=\left(x_{1}(t), \ldots, x_{n}(t)\right)^{T}$ is the solution of Eq. $(23)$. By the conditions $\left(A_{1}\right)-\left(A_{4}\right)$, we obtain

$$
\begin{aligned}
& L V_{i}(x(t)) \leq p\left|x_{i}(t)\right|^{p-1}\left\{-a_{i}(t)\left|x_{i}(t)\right|+\sum_{j=1}^{n}\left|a_{i j}(t)\right|\left|f_{j}\left(x_{j}(t)\right)\right|\right. \\
& \left.+\sum_{j=1}^{n}\left|b_{i j}(t)\right| \int_{-\infty}^{t}\left|p_{i j}(t-s)\right|\left|g_{j}\left(x_{j}(s)\right)\right| d s+\left|I_{i}(t)\right|\right\} \\
& +\frac{1}{2} p(p-1)\left|x_{i}(t)\right|^{p-2} \sigma_{i}^{T}\left(t, x_{i}(t)\right) \sigma_{i}\left(t, x_{i}(t)\right) \\
& \leq\left(-p a_{i}\left|x_{i}(t)\right|^{p}+\sum_{j=1}^{n} a_{i j} l_{j} p\left|x_{i}(t)\right|^{p-1}\left|x_{j}(t)\right|\right. \\
& +\sum_{j=1}^{n} b_{i j} k_{j} p\left|x_{i}(t)\right|^{p-1} \int_{0}^{\infty}\left|p_{i j}(s)\right|\left|x_{j}(t-s)\right| d s+I_{i} p\left|x_{i}(t)\right|^{p-1} \\
& \left.+\frac{1}{2} p(p-1)\left|x_{i}(t)\right|^{p-2} c_{i} x_{i}^{2}\right) h(t) \\
& \leq\left(-p a_{i}\left|x_{i}(t)\right|^{p}+\sum_{j=1}^{n} a_{i j} l_{j}\left(\left|x_{j}(t)\right|^{p}+(p-1)\left|x_{i}(t)\right|^{p}\right)\right. \\
& +\sum_{j=1}^{n} b_{i j} k_{j} \int_{0}^{\infty}\left|p_{i j}(s)\right|\left(\left|x_{j}(t-s)\right|^{p}+(p-1)\left|x_{i}(t)\right|^{p}\right) d s \\
& \left.+(p-1)\left|x_{i}(t)\right|^{p}+I_{i}^{p}+\frac{1}{2} c_{i} p(p-1)\left|x_{i}(t)\right|^{p}\right) h(t) \\
& =\left(\left\{-p a_{i}+\sum_{j=1}^{n} a_{i j} l_{j}(p-1)+\sum_{j=1}^{n} b_{i j} k_{j}(p-1) \int_{0}^{\infty}\left|p_{i j}(s)\right| d s\right.\right. \\
& \left.+(p-1)+\frac{1}{2} c_{i} p(p-1)\right\}\left|x_{i}(t)\right|^{p}+\sum_{j=1}^{n} a_{i j} l_{j}\left|x_{j}(t)\right|^{p} \\
& \left.+\sum_{j=1}^{n} b_{i j} k_{j} \int_{0}^{\infty}\left|p_{i j}(s)\right|\left|x_{j}(t-s)\right|^{p} d s+I_{i}^{p}\right) h(t) \\
& =\left(\sum_{j=1}^{n} p_{i j} V_{j}(x)+\sum_{j=1}^{n} \int_{0}^{\infty} q_{i j}(s) V_{j}(x(t-s)) d s+I_{i}^{p}\right) h(t), t \geq t_{0} .
\end{aligned}
$$


By Itô's formula, we obtain

$$
V_{i}(x(t))=V_{i}\left(x\left(t_{0}\right)\right)+\int_{t_{0}}^{t} L V_{i}(x(s)) d s+\int_{t_{0}}^{t} \frac{\partial V_{i}(x(s))}{\partial x} \sigma_{i j}(s, x(s)) d w(s) .
$$

Then we have

$$
E V_{i}(x(t))=V_{i}\left(x\left(t_{0}\right)\right)+\int_{t_{0}}^{t} E L V_{i}(x(s)) d s
$$

and for small enough $\Delta t>0$,

$$
E V_{i}(x(t+\Delta t))=V_{i}\left(x\left(t_{0}\right)\right)+\int_{t_{0}}^{t+\Delta t} E L V_{i}(x(s)) d s
$$

Thus, from (29), (30) and (31), we have

$$
\begin{aligned}
& E V_{i}(x(t+\Delta t))-E V_{i}(x(t)) \\
= & \int_{t}^{t+\Delta t} E L V_{i}(x(s)) d s \\
\leq & \int_{t}^{t+\Delta t}\left(\sum_{j=1}^{n} p_{i j} E V_{j}(x(s))+\sum_{j=1}^{n} \int_{0}^{\infty} q_{i j}(u) E V_{j}(x(s-u)) d u+I_{i}^{p}\right) h(t) d s,
\end{aligned}
$$

Then from (32), we obtain that

$$
D^{+} E V_{i}(x(t)) \leq\left(\sum_{j=1}^{n} p_{i j} E V_{j}(x(t))+\sum_{j=1}^{n} \int_{0}^{\infty} q_{i j}(s) E V_{j}(x(t-s)) d s+I_{i}^{p}\right) h(t) \text {. }
$$

Since $-(P+Q)$ is an $M$-matrix, then from Theorem 5.1, the solutions of (23) are uniformly bounded. By simply computing, Condition (4) is satisfied by Condition $\left(A_{1}\right)-\left(A_{3}\right)$. From Theorem 3.1, then there must exist an $\omega$-periodic Markov process. The proof is complete.

\section{Conclusion}

In this paper, we discuss a class of periodic Itô stochastic delay differential equations by using the properties of periodic Markov processes, and some sufficient conditions for the existence of periodic solution of the delay equations are given. As applications, we study the existence of periodic solution of periodic stochastic logistic equation and periodic stochastic neural networks with infinite delays, respectively. In our following papers, we will apply the existence theorem of periodic solution obtained in this paper to study the existence of periodic solution of periodic stochastic population system. 
Acknowledgments. The authors would like to thank the referees for their detailed comments and valuable suggestions which considerably improved the presentation of the paper.

\section{References}

[1] D. D. Bainov and V. B. Kolmanovskii, Periodic solution of stochastic functional differential equations, Math. J. Toyama Univ. 14 (1991), 1-39.

[2] L. E. Bertram and P. E. Sarachik, Stability of Circuits with randomly time-varying parameters, IRE. Trans. Circuit Theory, CT-6, Special supplement, 1959, 260-270.

[3] R. Z. Has'minskii, On the dissipativity of random processes defined by differential equations, Problemy Peredači Informacii 1 (1965), no. 1, 88-104.

[4] Stochastic Stability of Differential Equations, Sijthoff and Noordhoff, Maryland, 1980.

[5] K. Itô, On stochastic differential equations, Mem. Amer. Math. Soc. (1951), no. 4, 51 pp.

[6] K. Itô and M. Nisio, On stationary solutions of a stochastic differential equation, J. Math. Kyoto Univ. 4 (1964), no. 1, 1-75.

[7] D. Q. Jiang and N. Z. Shi, A note on nonautonomous logistic equation with random perturbation, J. Math. Anal. Appl. 303 (2005), no. 1, 164-172.

[8] D. Q. Jiang, N. Z. Shi, and X. Y. Li, Global stability and stochastic permanence of a non-autonomous logistic equation with random perturbation, J. Math. Anal. Appl. 340 (2008), no. 1, 588-597.

[9] V. B. Kolmanovskii and A. Myshkis, Introduction to the Theory and Application of Functional Differential Equations, London, 1999.

[10] X. X. Liao and X. R. Mao, Exponential stability and instability of stochastic neural networks, Stochastic Anal. Appl. 14 (1996), no. 2, 165-185.

[11] K. N. Lu and B. Schmalfuss, Invariant manifolds for stochastic wave equations, J. Differential Equations 236 (2007), no. 2, 460-492.

[12] X. R. Mao, Exponential Stability of Stochastic Differential Equations, Monographs and Textbooks in Pure and Applied Mathematics, 182. Marcel Dekker, Inc., New York, 1994.

[13] _ Stochastic Differential Equations and Applications, Horwood, Chichester, 1997.

[14] L. Y. Teng, L. Xiang, and D. Y. Xu, Existence-uniqueness of the solution for neutral stochastic functional differential equations, Rocky Mountain Journal of Mathematics (in press).

[15] D. Y. Xu, Y. M. Huang, and Z. G. Yang, Existence theorems for periodic Markov process and stochastic functional differential equations, Discrete Contin. Dyn. Syst. 24 (2009), no. $3,1005-1023$.

[16] D. Y. Xu and L. G. Xu, New results for studying a certain class of nonlinear delay differential systems, IEEE Trans. Automat. Control 55 (2010), no. 7, 1641-1645.

[17] B. G. Zhang, On the periodic solution of $n$-dimensional stochastic population models, Stochastic Anal. Appl. 18 (2000), no. 2, 323-331.

DingShi LI

School of Mathematics

SOUTHWEST JiAOTONG UNIVERSITY

Chengdu, 610031, P. R. China

E-mail address: lidingshi2006@163.com 
PERIODIC SOLUTIONS OF STOCHASTIC DELAY DIFFERENTIAL EQUATIONS 1181

\section{DAOYI XU}

Yangtze Center of Mathematics

SiCHUAN UNIVERSITY

Chengdu, 610064, P. R. China

E-mail address: dyxu@scu.edu.cn 